\title{
Molecular Neurobiology
}

National Cancer Institute

\section{Source}

National Cancer Institute. Molecular Neurobiology. NCI Thesaurus. Code C16873.

The branch of neurobiology that tries to understand the anatomy, physiology, and pathology of the nervous system by understanding the way that genes, proteins, and molecules interact. 